\title{
Comunicación, discursos, algoritmos, poder
}

institucionales.us.es/ambitos/

\author{
Miguel de Aguilera \\ Universidad de Málaga \\ deaguilera@uma.es
}

Aimiris Sosa

Universidad de Málaga

aimirissosa@uma.es

\section{Rocío de Aguilera \\ Universidad de Málaga \\ rocio.deaguileraper@gmail.com}

English Version: Communication, discourses, algorithms, power

Tras remontarse a los planteamientos de la Escuela de Frankfurt y las características del Análisis Crítico del Discurso, el autor sostiene que, si bien este tipo de debates no han desaparecido, en la actualidad el tema se contempla desde ópticas más complejas, al entrar en liza la sociedad digital en la que ha aumentado espectacularmente el número de agentes sociales que ejercen la comunicación pero lo hacen sobre narrativas de fondo en buena medida clásicas, al tiempo que aparecen otras más novedosas no exentas de críticas como es el caso de las redes sociales. Todo ello ha devenido en una gran cantidad de información que precisa ser ordenada, es en este momento cuando el algoritmo representa una herramienta pensada para tal fin, si bien el hecho también ofrece una situación preocupante para la actividad puramente Humana.

Palabras clave: comunicación, utopía, distopía, digitalización, algoritmo.

Abstract: After going back to the approaches of the Frankfurt School and the characteristics of the Critical Discourse Analysis, the author argues that, while this type of debate has not disappeared, currently the subject is seen from more complex perspectives, when the digital society enters into which has dramatically increased the number of social agents that they exercise communication but they do it on background narratives
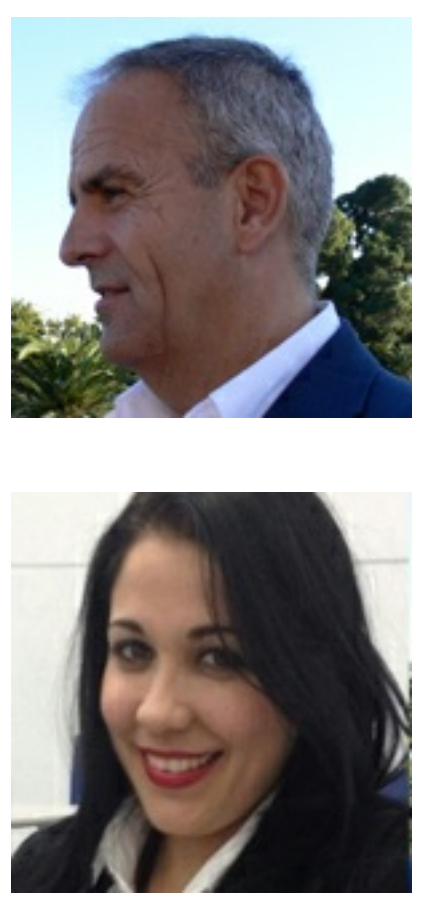
in good classic measures, while other newer ones not exempt from criticism as is the case of social networks. All this has become into a large amount of information that needs to be ordered, is at this moment when the algorithm represents a tool designed for that purpose, although the fact also offers a worrisome situation for purely human activity. 
Keywords: communication, utopia, dystopia, digitalization, algorithm.

\section{Introducción}

A lo largo del último cuarto del siglo XIX y de todo el siglo XX las sociedades más avanzadas vieron el nacimiento y desarrollo de una serie de medios de comunicación. Que desde su mismo nacimiento estuvieron estrechamente vinculados, por lo general, con las élites sociales -con los sectores dominantes en las esferas económica, política y cultural-, como bien quedó ya establecido desde los trabajos pioneros de la Escuela de Frankfurt. Por más que unas fueran públicas y otras privadas, las que pronto se conocieron como industrias de la cultura constituyeron en sí mismas, en su conjunto, un buen -aunque a menudo arriesgado- negocio, y además sirvieron a otros fines económicos y a dar visibilidad a ciertos fenómenos y personas. Entre las peculiaridades más importantes de ese sistema de comunicación se encuentra su capacidad para, apelando a la razón y/o a las emociones -según el medio, el lenguaje empleado o el tipo de contenido-, dar cierto sentido a los mensajes, proponiendo con ello una visión de la realidad que, de asentarse entre la población, habría de contribuir a la consolidación de la hegemonía social de ciertos sectores.

De modo que ese sistema de comunicación constituyó, prácticamente desde su mismo nacimiento, un excelente escenario para la confluencia de los poderes económicos y políticos en el ejercicio del poder simbólico. Para completar la "producción de verdad" (Foucault, 2004, 2014), esencial para el ejercicio de ese poder, esas mismas élites propiciaron la elaboración de interpretaciones de los fenómenos comunicacionales acordes, en términos generales, con sus intereses e insertas en más amplios discursos (de Aguilera, 2004, 2007). Y es que los medios de comunicación ejercen una notable función mediadora entre la realidad y nuestro conocimiento e interpretación de ella, condicionando el despliegue de nuestras acciones en los distintos escenarios sociales. De ahí que los sectores dominantes se esforzasen por lograr el control "del texto y del contexto" (Van Dijk, 1999: 28), buscando orientar el sentido de las comunicaciones y al mismo tiempo establecer un sistema interpretativo que lo legitimase. $Y$ esa forma de interpretar las comunicaciones -en un modelo, de fuerte aroma positivista e informacional, de comunicación lineal, dirigida y con propósitos de influencia de las élites sobre las "masas"alcanzó también una pronta y duradera posición hegemónica entre los profesionales y la población en general; entre quienes ha llegado a asentarse en su sistema de creencias básicas. Aunque también sea verdad que otros sectores de la población han pugnado con los dominantes por dar sentido a las comunicaciones, proponiendo asimismo modelos diferentes de interpretación -que en algunos casos han alcanzado también gran difusión y aceptación-. Y es que las comunicaciones ocupan desde hace más de un siglo una posición de primera importancia en nuestras sociedades. En consecuencia, son muchos y variados los actores sociales que han propuesto ideas y debatido su marco interpretativo. $Y$ esos debates, centrados en general en torno a los mecanismos que median en el ejercicio del poder simbólico, se integran en el contexto de más amplios discursos que, con mucha frecuencia -a veces parece tener un carácter cíclico-, siguen una orientación bien utópica o bien distópica.

En la actualidad no han desaparecido, por supuesto, esos medios de comunicación ni disminuido la importancia del ejercicio del poder simbólico a través suyo; ni han 
desaparecido tampoco las interpretaciones ni los debates que sobre ellos se desarrollaron. Pero sí es verdad que tanto los medios como sus interpretaciones se integran hoy en un sistema más complejo, que ocupa una posición de primera importancia en muchas facetas de la vida en sociedad. Pues es de sobra conocida la actual revolución que se da en el sistema comunicativo -asociada entre otros factores a la digitalización y a los distintos usos que les dan numerosos actores-, en el que coinciden viejos y nuevos medios, así como el papel mediador de primera importancia que esos medios y formas de comunicación representan en nuestra vida, tanto a título individual cuanto colectivo. Como es bien sabido, en ese nuevo escenario comunicacional ya no sólo participan las élites y los profesionales de las comunicaciones, sino también muchos más actores sociales, de condición e índole diversa -si bien los cauces de que disfrutan para la difusión de los mensajes y su alcance siga siendo muy desigual-. En general, los distintos actores interpretan su participación en ese contexto a luz de las circunstancias que les son propias, integrando esas interpretaciones en ciertos discursos construidos con mayor o menor coherencia. Muchos actores, pues, y muchas "voces" proponiendo interpretaciones más o menos variadas, aunque por lo general atravesadas por las grandes narrativas de fondo.

Y es que la mayoría de las explicaciones ofrecidas sobre los cambios habidos en el campo de la comunicación -y más extensamente en el conjunto de nuestras vidas- se integran en algunas narraciones de amplio calado, por cierto ampliamente difundidas y asumidas por la población, que cuentan con diferentes elementos narrativos, algunos hondamente arraigados en nuestras culturas. Así, refiriéndose a las posibilidades de uso de ciertos elementos tecnológicos a los que se ha atribuido algún carácter mesiánico (el ordenador, internet, el teléfono móvil), han alcanzado gran difusión narrativas como la que contribuyó notablemente a elaborar Nick Negroponte, de pronunciada orientación mítica y utópica. Que, entre otras cuestiones, plantea internet como una "nueva frontera", como un espacio a colonizar por nuevos actores sociales, como un ágora que permita profundizar el ejercicio de la democracia, como un escenario ideal para emprender nuevos negocios. $Y$ obviamente no le faltaba alguna razón al interpretar e, incluso, predecir ciertos fenómenos. Algo similar puede decirse de otras interpretaciones, de índole variada, en cuyo marco se incluyen expresiones tales como "cultura participativa" (Jenkins), "inteligencia colectiva" (Lévy), "remix" (Lessig), "autocomunicación de masas" (Castells), "prosumer" (Toffler) y "empoderamiento" [1]. Términos que, por cierto, han alcanzado gran popularidad y se refieren a fenómenos singulares y llamativos de la realidad, si bien se integran en discursos que enfatizan mucho esos rasgos de la realidad.

Aunque esas narraciones de signo utópico hayan ocupado durante bastantes años una posición destacada en nuestra forma de interpretar el rumbo que siguen en la actualidad las comunicaciones, conviven sin embargo con otras narraciones de signo contrario. Incluso, en los últimos años van adquiriendo cada vez mayor relevancia y difusión los trabajos que ponen de manifiesto los aspectos negativos de esta revolución comunicacional y de algunos de sus usos más destacados, proyectando sobre ellos, cuando menos, una visión pesimista, si no abiertamente distópica. Y ello, entre otras razones, porque en estos renovados escenarios de la acción humana se desarrollan con notable eficacia las prácticas basadas en la "contra-utopía tecnocrática", esto es, en la visión de la naturaleza humana y del mundo apoyada en supuestos radicalmente opuestos a los de las utopías humanísticas y que en la actualidad busca orientar la innovación 
tecnológica, el "progreso científico y técnico hacia el crecimiento económico" (Manuel Martín Serrano , 2014: 19) -así como justificar en el nivel de las ideas esa orientación-. Y es que el ciberespacio es un escenario no clausurado en torno de un solo fin, un espacio multipropósito donde se desarrollan las acciones, con fines diferentes y de índole y signo muy variados, desplegadas por los diversos actores sociales. Un escenario privilegiado en nuestros días, también, para el desarrollo de una fuerte pugna entre la búsqueda de una mayor autonomía -individual y colectiva- y el intento de su control -colonización- por los poderes políticos y económicos. Así, en él concurren muchos factores, condiciones y agentes, en un contexto que muta y se transforma, y en el que ciertos actores están alcanzando una nítida posición dominante. Por ejemplo, Google, Facebook, Amazon y algunos otros, que por cierto mantienen determinadas prácticas con enorme eficacia aunque sus discursos se apoyen con frecuencia en componentes utópicos -lo que es un signo de nuestros tiempos-.

Así, en el ámbito económico se multiplican las denuncias y debates sobre el "trabajo inmaterial", el "capitalismo cognitivo", el "capitalismo de plataformas" o el "prosumer capitalism", entre otras cuestiones. Estas expresiones subrayan determinados rasgos de las actividades económicas, y los integran en ciertos marcos interpretativos. De modo que algunos autores llegan a concebir ahora el ciberespacio, en efecto, como una nueva frontera pero, como la del "Far West", no sometida al imperio de leyes y normas que debieran ordenar las interacciones humanas que en ese escenario tienen lugar.

Las redes sociales, entre las que Facebook desempeña un papel muy relevante, resultan asimismo muy ilustrativas de esta evolución en las narraciones explicativas así como en su consecuente percepción pública. Pues durante años los rasgos en los que más énfasis se puso en esos discursos han sido que su uso aumenta la sociabilidad, además de constituir una nueva "ágora", un nuevo espacio donde compartir ideas y formar opiniones, una poderosa herramienta para el cambio social y el empoderamiento ciudadano, un espacio propicio para el activismo digital. Las alusiones, tan frecuentes durante algún tiempo, al "periodismo ciudadano" y la "primavera árabe" -que arranca en Túnez en diciembre de 2011-, reflejan bien esos discursos esperanzados, con sabor utópico, que durante un tiempo han orientado la percepción pública de esas redes.

Aunque sigan abundando en nuestros días los trabajos que resaltan las aportaciones positivas de las redes a distintos ámbitos de la vida, sin embargo, son cada vez más comunes los que adoptan un punto de vista negativo sobre los usos de esas redes (de Aguilera y Casero, 2018). Se multiplican los ejemplos. En el caso de Facebook, y ya que su acción concierne sobre todo a la interacción entre individuos, pero también a la distribución de informaciones, hay quienes la critican vivamente por razón del tipo de sociabilidad que promueve y de los estímulos psicológicos en que se apoya-en especial, "refuerzos" asociados al reconocimiento de la visibilidad-. Pero la mayor oleada de críticas que recibe responde sobre todo a su uso en el desarrollo de estrategias de manipulación de las opiniones públicas mediante la difusión de informaciones deformadas o directamente falsas. Como es bien sabido, Facebook cuenta con más de 2000 millones de personas integradas en su comunidad de usuarios y es líder en redirigir a sus lectores hacia contenidos informativos. Su responsabilidad como medio de distribución de noticias es elevada. Entre otras, respecto de las emitidas en el marco de campañas de 
desinformación o con otros fines persuasivos por entidades y personas especializadas en ello -que conocen bien y saben usar en su beneficio los algoritmos y otras características de las redes- y radicadas en países tales como Rusia, Filipinas o Corea del Norte, o en los mismos EE.UU de América, entre otros.

Con independencia de los efectos que esas campañas puedan haber tenido entre las audiencias alcanzadas, es indiscutible que a través suyo se han difundido dichas informaciones. Pues Facebook, como otras redes, no crea contenidos, pero sí los ordena y contribuye a su difusión. Así, la difusión de informaciones falsas u otras de contenido claramente censurable para la mayoría de la población afecta a su credibilidad y a otros factores fundamentales para la confianza del usuario en esa red, que sobre todo opera en un espacio semi-público. De aquí que esa empresa comunicacional decidiese primero realizar una labor editorial para seleccionar las noticias que se difundieran. Que atribuyó inicialmente a un grupo de periodistas, para pasar después a confiar esa labor a un algoritmo. Desde entonces Facebook ha tomado una serie de medidas con el fin de recuperar su credibilidad y perfilar mejor su modelo de negocio, que incluye reducir el número de informaciones difundidas a través de esa red ${ }^{[2]}$ y atribuir a ese algoritmo -así como a su posterior mejora basada en el aprendizaje automático- una serie de funciones que tradicionalmente han desempeñado las personas -tales como gatekeeping y curationsirviéndose de las herramientas brindadas por el contexto cultural en cuyo seno ejerciesen sus funciones. Entre ellas, clasificar, filtrar, priorizar, recomendar y decidir, incluso, qué medios informativos resultan más fiables para los usuarios de la red.

Y es que en sociedades como la nuestra, en alta medida digitalizadas, las enormes cantidades de datos generadas en los distintos escenarios de nuestra vida cotidiana, así como ciertas tendencias tecnológicas, están propiciando que apoyemos muchas de nuestras tradicionales actividades en esos algoritmos. Actividades que se extienden, por ejemplo, desde la selección de la pareja adecuada hasta el filtrado y la recomendación de contenidos culturales con los que gestionar nuestras emociones o con los que informarnos de lo que acontece. $Y$ es que los algoritmos, en un número creciente de escenarios, condicionan lo que conocemos, cómo lo conocemos, lo que descubrimos, lo que experimentamos; y lo que decidimos, no sólo en los escenarios de nuestra vida privada sino también en muchos ámbitos institucionales u organizacionales. De aquí que algunos autores hablen ya, con preocupación, de una "cultura algorítmica" (Striphas, 2015), en la que esos elementos técnicos complementan -reemplazándonos cada vez más- ciertas actividades culturales humanas. Y también, entre cuestiones diversas, del "efecto burbuja" que la mediación algorítmica produce, pues reduce o elimina la exposición a puntos de vista diferentes de aquellos en los que estamos inicialmente.

Los algoritmos constituyen un nuevo elemento mediador en nuestro entorno, que condiciona nuestra percepción de lo que nos rodea y nuestra capacidad de interactuar con ello. Este nuevo elemento mediador, por supuesto, no es ajeno a las formas del poder propias de nuestra sociedad. Pues, más allá de su evidente vínculo con los nuevos poderes económicos, a través suyo se desarrolla y expresa el poder. Y es que, aunque formen parte de esas "tecnologías enigmáticas" (Pasquale, 2015) propias de nuestra época, de ellas sabemos bastante como para afirmar que los algoritmos no son simples líneas de códigos, sino que se integran en determinados procesos sociales a los que 
sirven las tecnologías de las que cada algoritmo forma parte. Los procesos de su elaboración obedecen a la confluencia de varias lógicas profesionales e incluyen toda una serie de criterios. Entre otros, tal y como afirmaba Tania Bucher al examinar el algoritmo de Facebook "algorithm is not merely modelled on a set of pre-existing cultural assumptions, but also on anticipated or future oriented assumptions about valuable and profitable interactions that are ultimately geared towards commercial and monetary purposes" (Bucher, 2012: 7).

Así que, basados en la obediencia a ciertos criterios que siguen una lógica empresarial, y sobre todo en escenarios con enormes volúmenes de datos, los algoritmos realizan funciones que antes realizaba en exclusiva el ser humano empleando herramientas proporcionadas por su entorno cultural. Y así, al integrarse en las prácticas de nuestra vida cotidiana, contribuye a producir y a dar forma a lo que conocemos y experimentamos.Pero además, los algoritmos se nos presentan en el marco de discursos relativos a la eficiencia, la neutralidad, la transparencia y una calculable y confiable objetividad. Como parte de una racionalidad más amplia, inserta en ese aparato de conocimiento a través del que el poder es promulgado (Foucault, 2004: 34).

El poder de los algoritmos -la expresión del poder a través suyo- se ejerce, pues, de dos modos principales: por un lado, mediante la "verdad" a la que se adhiere el algoritmo, es decir a través de la noción misma de algoritmo,del sistema de ideas y creencias de las que el algoritmo forma parte y que se enuncia mediante ciertos discursos; por otro lado, mediante la "verdad" que el algoritmo produce al integrarse en diversas prácticas de la vida cotidiana, a las que contribuye a dar forma. Esto es, en términos más queridos por Foucault, mediante la producción de verdad y su enunciación discursiva.

\section{Referencias}

Beer , D. (2017). The social power of algorithms.Information, Communication \& Society, 20:1, 1-13, DOI: 10.1080/1369118X.2016.1216147

Bucher, T. (2012). Want to be on top? Algorithmic power and the threat of invisibility on Facebook. New Media \& Society, 14, 1164-1180. doi:10.1177/1461444812440159

de Aguilera, M. (2004). Tomar la cultura popular en serio. Comunicación, 2, 147-58.

de Aguilera, M. (2007). Les modèles de la communication et leur objet d'étude.Communication \& Organisation, 30, 111-26.

de Aguilera, M., Casero Ripollés, A. (2018). ¿Tecnologías para la transformación? Los medios sociales ante el cambio político y social. ICONO 14 16, n. 1, p. 1-21.Doi:

10.7195/ri14.v16i1.1162

Foucault, M. (2004). Society must be defended: Lectures at the collège de France, 197576. London:Penguin.

Foucault, M. (2014). On the government of the living: Lectures at the collège de France 1979-1980.Basingstoke: Palgrave Macmillan. 
Martín Serrano, M. (2014). La globalización: un espacio y un tiempo deconfrontación entre opciones humanizadoras y deshumanizadoras. Telos, 98, 14-23.

Pasquale, F. (2015). The black Box society: The secret algorithms that control money and information. Cambridge, MA: Harvard University Press.

Striphas, T. (2015). Algorithmic Culture. European Journal of Cultural Studies, 18(4-5) 395412

Van Dick, T. (1999). El análisis crítico del discurso. Anthropos, 186, 23-26.

[1] Idea desarrollada por diversos autores en contextos diferentes y, si bien vinculada de raíz con el poder y sus ejercicios, sin embargo, ceñida en la práctica comunicacional a otorgar visibilidad y voz a algunos nuevos actores.

[2] Lo que representa un grave problema para las empresas informativas especializadas en el uso de las redes sociales para lograr su difusión.

\section{BREVE SEMBLANZA DE LOS AUTORES:}

Miguel de Aguilera es Catedrático de Comunicación Audiovisual y Publicidad en la Universidad de Málaga, en la que fue Decano de su Facultad de Ciencias de la Comunicación y Director General de Comunicación e Información. Autor de numerosas publicaciones, con editoriales y revistas de Alemania, Reino Unido, Italia, Francia, Estados Unidos, México, Brasil y Austria, entre otros países. Su investigación se especializa, en general, en la cultura digital, en la cultura popular y en sus usuarios.

Aimiris Sosa es doctoranda del Programa de Doctorado Interuniversitario en Comunicación de las Universidades de Cádiz, Huelva, Málaga y Sevilla. Contratada FPU del Ministerio de Educación, Cultura y Deporte de España. Licenciada en Periodismo por la Universidad Central "Marta Abreu" de Las Villas, Cuba. Máster en Gestión Estratégica e Innovación en Comunicación por la Universidad de Málaga.

Rocío de Aguilera es publicista y periodista. Consultora independiente especializada en marketing digital, colabora con agencias de comunicación y organizaciones no gubernamentales de varios países.

- Recibido: 03/03/2018

- Aceptado: 09/03/2018

Ámbitos. Revista Internacional de Comunicación, n.40, edición de primavera, 2018. 